\title{
COVID-19 pandemic year in a sample of Polish myasthenia gravis patients: an observational study
}

\author{
Łukasz Rzepiński ${ }^{1,2}$, Monika Zawadka-Kunikowska ${ }^{3}$ \\ ${ }^{1}$ Department of Neurology, $10^{\text {th }}$ Military Research Hospital and Polyclinic, Bydgoszcz, Poland \\ ${ }^{2}$ Sanitas-Neurology Outpatient Clinic, Bydgoszcz, Poland
}

${ }^{3}$ Department of Exercise Physiology and Functional Anatomy, Ludwik Rydygier Collegium Medicum in Bydgoszcz Nicolaus Copernicus University in Torun, Bydgoszcz, Poland

\begin{abstract}
Aim of the study. Coronavirus disease 2019 (COVID-19) incidence, mortality, recovery and hospitalisation rates vary in different countries. This study aimed to present the clinical characteristics of a sample of unvaccinated Polish myasthenia gravis (MG) patients during the first year of the COVID-19 pandemic, taking into account the number of MG exacerbations, a detailed description of the severe acute respiratory syndrome coronavirus 2 (SARS-CoV-2) infection course, and the need to modify immunosuppressive therapies.

Clinical rationale for the study. To assess the impact of the first COVID-19 pandemic year on MG course in a sample of unvaccinated patients.

Materials and methods. A retrospective observational study involving 30 unvaccinated Polish MG patients consulted in a neurological outpatient clinic on 11-31 March, 2020 (baseline) and 11-31 March, 2021 (endpoint).

Results. During the period of evaluation, exacerbation of MG requiring hospitalisation was reported in 11 patients. Among them, four were treated with intravenous immunoglobulin and another six required plasma exchange. In the study group, COVID-19 was identified in 10 patients. Of them, seven experienced a mild course of SARSCoV-2 infection with spontaneous recovery. In the remaining three patients, both MG exacerbation and SARS-CoV-2 infection were reported. These patients experienced MG exacerbation in the preceding month or concurrently with COVID-19 and were aged over 50 years. Due to the SARS-CoV-2 infection, they required antibiotic and oxygen therapy, and hospitalisation was necessary in the case of two obese patients. None of the patients died due to COVID-19, and nor did any require discontinuation of immunosuppressive therapies during the study period. In total, 12 patients in the study group experienced neither MG exacerbation nor SARS-CoV-2 infection.

Conclusions. In the presented sample of Polish MG patients, favourable outcomes of COVID-19 were observed. Further studies are needed to evaluate the reliable course of COVID-19 taking into account international differences, the types of treatment applied, and the ratio of vaccinated to unvaccinated MG patients.
\end{abstract}

Key words: myasthenia gravis, COVID-19, SARS-CoV-2, pandemic, immunosuppression

(Neurol Neurochir Pol 2022; 56 (1):61-67)

\section{Introduction}

Myasthenia gravis (MG) is the most common acquired autoimmune disease targeting the neuro-muscular junction (NMJ) [1]. The prevalence of MG in Poland is estimated at $22.65 / 100,000$, with an annual incidence of 2.36/100,000 [2].
The disease is caused by autoantibodies that bind to acetylcholine receptors (AChR), muscle-specific kinase (MuSK), or to other AChR-related postsynaptic membrane molecules of the NMJ [1]. Dysfunction of neuromuscular transmission results in fatigable muscle weakness, which fluctuates over time. The involvement of respiratory muscles can lead to myasthenic

Address for correspondence: Łukasz Rzepiński, Department of Neurology, 10th Military Research Hospital and Polyclinic, Bydgoszcz, Poland, e-mail: luk.rzepinski@gmail.com

Received: 27.04.2021 Accepted: 6.07.2021 Early publication date: 3.08.2021

This article is available in open access under Creative Common Attribution-Non-Commercial-No Derivatives 4.0 International (CC BY-NC-ND 4.0) license, allowing to download articles and share them with others as long as they credit the authors and the publisher, but without permission to change them in any way or use them commercially. 
crisis, a life-threatening condition that requires intensive care and respiratory support [3]. On the other hand, fatigue and muscle weakness as well as respiratory failure are cardinal symptoms of Coronavirus disease 2019 (COVID-19) [4, 5]. Therefore, it can be very challenging to distinguish between these two conditions as well as to diagnose MG exacerbation during a severe acute respiratory syndrome coronavirus 2 (SARS-CoV-2) infection [6]. Moreover, it has been shown that pre-existing neurological disorders are associated with a worse prognosis and a high COVID-19 fatality rate [7]. The first case of SARS-CoV-2 infection in Poland was confirmed on 4 March 2020. On 11 March 2020, the World Health Organisation declared the COVID-19 outbreak to be a pandemic [8]. Even after more than a year of the pandemic, data on its impact on MG course is still limited.

Generally, MG patients may be at higher risk of unfavourable COVID-19 outcomes due to multiple issues. Firstly, patients with baseline respiratory muscle weakness as well as those using immunosuppressive therapy may be more severely affected by SARS-CoV-2 than their healthy peers. Secondly, viral infection itself, or exposure to some antibiotics (e.g. fluoroquinolones, macrolides, aminoglycosides) for bacterial superinfection, may trigger MG exacerbation [1]. Another issue is the limited access to systematic MG severity assessment by the attending neurologist and modification of the treatment regimen. However, the available data has shown highly variable MG reactions to COVID-19 that were independent of the viral infection's severity [9]. Some reports have described MG exacerbations during COVID-19, while others have shown a stable MG course throughout this infection [10, 11]. Moreover, COVID-19 incidence, mortality, recovery and hospitalisation rates vary greatly between different countries $[12,13]$. Until now, the course of SARS-CoV-2 infection in Polish MG patients has not been described.

Therefore, we aimed to present the clinical characteristics of a sample of Polish MG patients in the first year of the COVID-19 pandemic, taking into account the number of MG exacerbations, a detailed description of the SARS-CoV-2 infection course, and the need to modify immunosuppressive therapies.

\section{Clinical rationale for the study}

It is highly desirable to obtain data on the SARS-CoV-2 infection course in patients with rare autoimmune diseases. MG is classified as a rare autoimmune disease with geographically diverse prevalence and incidence, partly due to variations in demographics between countries [1]. Therefore, assessing the impact of the first year of the COVID-19 pandemic on unvaccinated Polish MG patients seems to be valuable from the clinician's perspective.

\section{Materials and methods}

This observational retrospective study included 30 Polish MG patients consulted in a neurological outpatient clinic on 11-31 March, 2020 (baseline) and 11-31 March, 2021 (endpoint).
The analysed clinical and demographic parameters included: age, sex, MG type and severity, MG duration, presence of antibodies against AChR or muscle-specific kinase (MuSK), occurrence of MG exacerbations or myasthenic crisis, diagnosis of SARS-CoV-2 infection, comorbidities, and the type of treatment applied for both MG and COVID-19 if applicable. The diagnosis of MG was established on the basis of clinical features (fluctuating weakness of ocular and/or extraocular muscles) and at least one of the following criteria: a positive test for AChR or MuSK autoantibodies, postsynaptic dysfunction of the NMJ confirmed by repetitive stimulation or single fibre electromyography, and/ or a clinical response to cholinesterase inhibitors.

A COVID-19 diagnosis was confirmed by a positive nasopharyngeal swab polymerase-chain-reaction (RT-PCR) test for SARS-CoV-2. With regard to the clinical disease type, ocular MG (signs restricted to the ocular muscles) or generalised MG (involvement of extraocular muscles) were distinguished [1]. Disease severity was assessed using the Myasthenia Gravis Foundation of America (MGFA) classification that separates patients into five groups. MGFA classes involve: pure ocular weakness (class I), mild-generalised weakness (class II), moderate-generalised weakness (class III), severe-generalised weakness (class IV), and intubation with or without mechanical ventilation (class V). Within the generalised MG types, patients are subcategorised as class A (predominant limb/axial muscles involvement) or class B (predominant oropharyngeal/ respiratory muscles involvement) [14]. MG exacerbation was defined as the presence of new, or worsening of previously reported, muscle weakness lasting more than 24 hours unrelated to fever and/or infection resulting in a shift between class $A$ and $B$ and/or an increase in the MGFA classification by at least one class. The worsening of symptoms within 30 days was considered to be a single exacerbation. Myasthenic crisis was characterised by worsening of muscle weakness leading to respiratory failure requiring intubation and mechanical ventilation [15]. Rapid worsening of MG symptoms that, in the opinion of the treating physician, could lead to crisis in the short term, was classified as an impending myasthenic crisis [16].

Thymic abnormalities were assessed based on computed tomography (CT) scan results and available histology findings. All patients were tested for the presence of serum anti-AChR antibodies by an enzyme-linked immunosorbent assay (ELI$\mathrm{SA}$ ), and values $\geq 0.4 \mathrm{nmmol} / \mathrm{L}$ were considered abnormal. IgG4 antibodies against MuSK were measured by ELISA in subjects lacking anti-AChR antibodies.

This study was approved by the Bioethical Committee of Collegium Medicum in Bydgoszcz, Nicolaus Copernicus University in Torun. All subjects participated voluntarily and gave their written informed consent.

\section{Results}

The baseline demographic and clinical characteristics of the study group are set out in Table 1. 
Table 1. Baseline demographic and clinical characteristics of study cohort

\begin{tabular}{|c|c|}
\hline & MG patients \\
\hline Number of subjects (n) & 30 \\
\hline Sex (Male/Female) & $5 / 25$ \\
\hline Age (years) & $42.3 \pm 12.0$ \\
\hline Disease duration (years)[range] & $9.6 \pm 7.2$ \\
\hline Age at first symptoms (years) & $34.5 \pm 11.3$ \\
\hline \multicolumn{2}{|l|}{ Type of MG } \\
\hline Ocular & $8(26.7 \%)$ \\
\hline Generalised & $21(70 \%)$ \\
\hline Seropositivity to AChR antibodies, n (\%) & $23(76.7 \%)$ \\
\hline Seropositivity to MuSK antibodies & $1(3.3 \%)$ \\
\hline Double-seronegative MG & $6(20 \%)$ \\
\hline Thymic pathology (\%) & $15(50 \%)$ \\
\hline Thymectomy, n (\%) & $11(36.7 \%)$ \\
\hline Thymoma & $1(3.3 \%)$ \\
\hline \multicolumn{2}{|l|}{ Disease severity (MGFA), $n(\%)$} \\
\hline Asymptomatic & $1(3.3 \%)$ \\
\hline Class I & $8(26.7 \%)$ \\
\hline Class II A & $14(46.7 \%)$ \\
\hline Class III A & $6(20 \%)$ \\
\hline Class III B & $1(3.3 \%)$ \\
\hline \multicolumn{2}{|l|}{ Type of treatment } \\
\hline Cholinergic therapy & $29(96.7 \%)$ \\
\hline Corticosteroids & $20(66.7 \%)$ \\
\hline Non-steroidal immunosuppressants & $15(50 \%)$ \\
\hline
\end{tabular}

- Myasthenia Gravis Foundation of America classification

In the presented cohort, none of the MG patients received a COVID-19 vaccine. In total, 12 patients experienced neither MG exacerbation nor SARS-CoV-2 infection. Moreover, these patients did not require modification of MG treatment regimen, and the severity of the disease as assessed by MGFA classification did not change during the study period. Exacerbation of $M G$ requiring hospitalisation was reported in 11 patients, 10 females $(\mathrm{F})$ and one male $(\mathrm{M})$. In all women, the disease exacerbation was not related to COVID-19 and the treatment regimen consisted of intravenous immunoglobulin (IVIG) infusions (four patients) or plasma exchange (PE) (six patients). Conversely, the MG exacerbation affecting one man in this subgroup was associated with a developing SARS-CoV-2 infection. Overall, COVID-19 was identified in 10 patients (nine $\mathrm{F}$, one $\mathrm{M}$ ), including eight with anti-AChR antibodies and two double-seronegative patients. Of these eight, seven experienced a mild course of SARS-CoV-2 infection with spontaneous recovery. These patients did not require hospitalisation, the use of antiviral drugs, or a change in MG treatment regimen. They did not experience myasthenia gravis exacerbation or bacterial superinfection. All were non-smokers without obesity or other comorbidities. The detailed demographic and clinical characteristics of this subgroup are set out in Table 2. In four patients (cases 1, 3, 4 and 5), SARS-CoV-2 infection was confirmed in other family members. Interestingly, these patients reported a less severe course of COVID-19 with a shorter recovery time than their closest relatives who experienced bacterial superinfection and required antibiotics.

In the remaining MG patients infected with SARSCoV-2 (cases 8, 9, and 10, described below), the course of COVID-19 was not self-limiting, requiring the use of oxygen therapy and antibiotics due to bacterial superinfection.

None of the patients died due to COVID-19, and nor did any require discontinuation of immunosuppressive therapies during the period of evaluation.

\section{Case 8}

A 53-year-old non-smoking woman with non-thymoma, generalised AChR (+) MG of 22 years' duration experienced disease exacerbation in January 2021. For this reason, she was hospitalised and IVIG infusions were used, with clinical improvement (MGFA IV A- > MGFA IIIA). Two weeks after discharge, the patient developed symptoms of SARS-CoV-2 infection in the form of fever, headache, cough, myalgia and shortness of breath. At that time, the patient was obese (BMI $=31.2$ ) and was receiving delfazacort ( $24 \mathrm{mg} /$ day), mycophenolate mofetil (2,000 $\mathrm{mg} / \mathrm{d})$, and pyridostigmine ( $540 \mathrm{mg} /$ day). The patient was admitted to the COVID ward where chest CT revealed patchy ground glass opacities in the right lung with normal arterial blood gas results. Empirical antibiotic therapy (ceftriaxone $2 \mathrm{~g}$ intravenously) and non-invasive respiratory support (nasal high flow cannula) were implemented, resulting in clinical improvement after 14 days. During COVID-19, the treatment regimen for $\mathrm{MG}$ was not changed. At the neurological consultation seven days after discharge, the severity of MG was classified as MGFA IIIA.

\section{Case 9}

A 52-year-old non-smoking woman with a history of asthma and overweight $(\mathrm{BMI}=26.8)$, had been diagnosed with generalised AChR (+) MG in 2018. Chest CT performed at the diagnostic stage did not reveal persistent thymus or thymoma. During the year of the COVID-19 pandemic, the patient experienced two exacerbations of $M G$ requiring hospitalisation. In the first, in March/April 2020, PE was performed, replacing a total of $8,900 \mathrm{ml}$ of plasma (MGFA IVA- > MGFA IIA). Three weeks before her COVID-19 infection, the patient was admitted to the Department of Neurology due to impending myasthenic crisis. For this reason, therapeutic cycles of PE were performed, with a total of $8,300 \mathrm{~mL}$ of plasma replaced, resulting in an improvement from class IVA to class IIIA according to the MGFA classification. At the time of her SARS-CoV-2 infection, the patient was receiving ambenonium (50 mg/day), delfazacort (12 mg/day) and azathioprine 


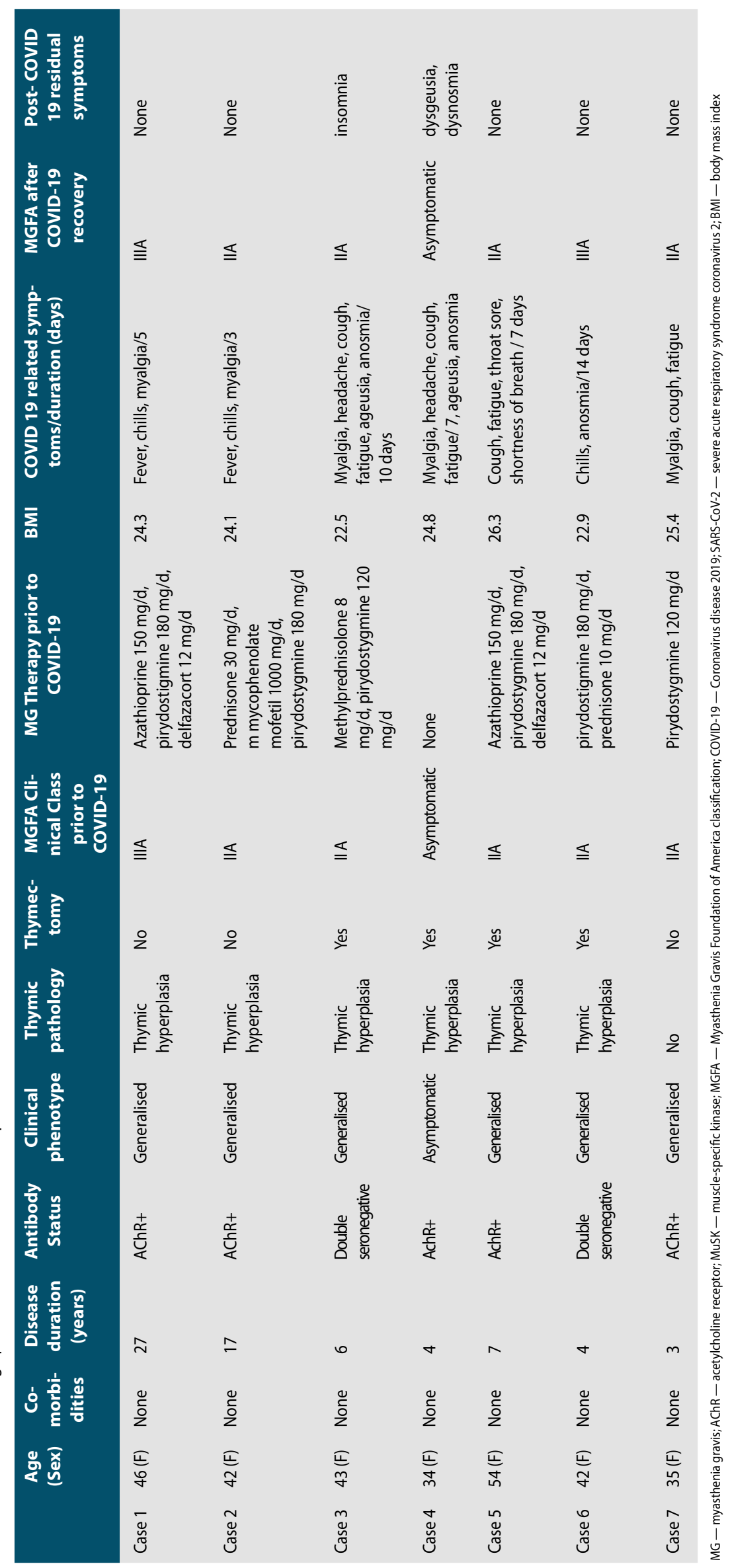


(150 mg/day). COVID-19 symptoms included headache, sore throat, runny nose, fever, chills, shortness of breath, myalgia, ageusia, and anosmia. Due to bacterial superinfection of the upper respiratory tract, empirical antibiotic therapy was applied (cefixime $400 \mathrm{mg} /$ day for 14 days). The patient required home oxygen therapy administered through a face mask for the first seven days of SARS-CoV-2 infection. After antibiotic therapy, the patient recovered and there was no need for hospitalisation or change in the treatment regimen for MG. The severity of MG after recovery from COVID-19 was assessed as IIIA according to the MGFA classification.

\section{Case 10}

A 62-year-old non-smoking man with a history of obesity (BMI 31.5), impaired glucose tolerance and nonalcoholic fatty liver disease. The first symptoms of MG, in the form of ptosis and diplopia, occurred in 2018. The diagnosis was established in the same year and additional tests performed at that time showed the presence of AChR antibody in the serum, and persistent thymus in chest $\mathrm{CT}$. Since diagnosis, the patient had had only ocular symptoms well controlled with pyridostigmine. On 19 January, 2021 the patient experienced bulbar symptoms, and therefore prednisone $40 \mathrm{mg} /$ day was added to the treatment regimen with only a partial clinical improvement (MGFA IVb- > MGFA IIIb). For this reason, the patient was qualified for admission to the Department of Neurology in his place of residence. At that time, the patient showed no signs of infection. However, a nasopharyngeal screening smear revealed the presence of SARS-COV-2 and chest CT showed inflammatory bilateral ground-glass opacification lesions covering $<10 \%$ of lung parenchyma. Over the following days, the patient developed fever, cough and dyspnoea requiring the use of high-flow oxygen therapy. In terms of treatment, steroid was maintained, and tocilizumab and ceftriaxone were added. Due to worsening anaemia, a total of four units of red blood cells were transfused. On 9 February, 2021, the patient was discharged with the severity of MG classified as MGFA IIb.

\section{Discussion}

This observational study aimed to describe a cohort of 30 Polish MG patients during the first year of the COVID-19 pandemic. In the study group, $36.7 \%$ of patients experienced MG exacerbation, and SARS-CoV-2 infection was detected in $33.3 \%$. Three patients (two $\mathrm{F}$ and one $\mathrm{M}$ ), representing $10 \%$ of the studied cohort, underwent both MG exacerbation as well as SARS-CoV-2 infection. These patients experienced MG exacerbation in the preceding month or concurrently with COVID-19, had antibodies against AChR, and were over 50 years of age. Due to the SARS-CoV-2 infection, they required antibiotic and oxygen therapy, and hospitalisation was necessary in the cases of two obese patients. The most severe course of COVID-19 was observed in the man in whom MG exacerbation coincided with the infection; until then, he had had only ocular symptoms and was not immunosuppressed. On the other hand, $70 \%$ of MG patients infected with SARSCoV-2 did not require the use of antiviral drugs, antibiotics and oxygen therapy. Among them were only women all nonobese: $28.6 \%$ had double seronegative MG, and $71.4 \%$ were immunosuppressed. Interestingly, none of these patients had a post-COVID-19 worsening of MG severity assessed using the MGFA classification. Importantly, no patient in the study group died due to COVID-19, and no patient had to discontinue immunosuppressive therapy or to change MG treatment regimen during infection.

Generally, COVID-19 course can be classified as asymptomatic, mild, moderate, or severe [17]. There is strong evidence that SARS-CoV-2 may be neuroinvasive and could cause a wide array of neurological symptoms and complications [18]. Farsalinos et al. suggested that COVID-19 may be a disease of the nicotinic cholinergic system. The authors hypothesised that SARS-CoV-2 may interact with the nicotinic $\mathrm{AChR}$ leading to dysregulation of the cholinergic anti-inflammatory pathway [19]. In this context, the presence of antibodies against $\mathrm{AChR}$ in $\mathrm{MG}$ patients may alter the incidence and course of COVID-19, something which should be examined in laboratory and clinical settings.

So far, there have been a few reports describing SARSCoV-2 infections in MG patients with a highly variable course and outcome. Some of these studies have included only patients who were hospitalszed $[20,21]$, while others also described outpatient management $[22,23]$. The percentage of patients who required hospitalisation and/or experienced MG exacerbation during SARS-CoV2 infection varies across studies, which is mainly due to the small size of the analysed groups. According to preliminary data from a global, physician-reported registry of $91 \mathrm{MG}$ patients infected with SARS-CoV-2, $63(69 \%)$ required hospitalisation, disease worsening or crisis of MG requiring rescue therapy (e.g. IVIG, PE, or steroids) was reported in 36 patients (40\%), complete recovery or discharge home was reported in $39(43 \%)$ patients, whereas $22(24 \%)$ patients died due to COVID-19 [22]. These figures are important because of the large number of MG patients diagnosed with COVID-19 to date, but they do not take into account international variations in the infection course. In a study by Solé et al. based on a French database for rare disorders, 34 out of 3,558 MG patients had COVID-19. Within the period of evaluation, 28 patients recovered from COVID-19, one remained affected, and five died. Interestingly, immunosuppressive therapies used for $M G$ were not risk factors for poorer outcomes, and only MGFA class $\geq$ IV before infection was associated with severe COVID-19 [24]. Compared to the presented cohort of Polish MG patients, the percentage of patients in the group of Solé et al. infected with SARS-CoV-2 was lower and fatalities occurred; this might reflect differences in the incidence and course of COVID-19 depending on the region of residence. Another important aspect influencing the course of SARS-CoV-2 infection in MG patients is the international differences in the number of residents vaccinated 
against COVID-19, a figure which should be included in all reports devoted to this issue.

Interestingly, the inter-study differences concern not only the course of COVID-19 in MG patients, but also the therapeutic approaches. Rein et. al presented three patients with generalised AChR + MG who developed SARS-CoV-2 infection. All of them had a favourable outcome despite continued immunosuppression, and only one patient experienced MG exacerbation requiring non-invasive ventilatory support with administration of hydroxychloroquine, lopinavir and ritonavir [23]. Anand et al. described COVID-19 course in five hospitalised and immunosuppressed MG patients (four with AChR antibodies and one with MuSK antibody). Of these, four patients had favourable outcomes and the authors decided to hold the home dose of mycophenolate mofetil in two of their patients [21]. In 2020, the International MG/COVID-19 Working Group provided guidance on $M G$ management during the SARS-CoV-2 pandemic. The expert panel recommended that MG patients should continue their current treatment and not to stop any existing medications unless specifically discussed and approved by their healthcare provider [25]. These recommendations have been successfully applied in the presented sample of Polish patients, in which no patient has required a change in their MG treatment regimen. It is worth noting that there is one case report describing an immunocompromised Polish MG patient who did not become infected despite close contact with her relatives who developed mild COVID-19 [26].

The main limitation of our study is the small number of patients and the short duration of follow-up, which makes it impossible to determine the long-term consequences of SARS-CoV-2 infection. Therefore, data from large registries are needed to present a reliable COVID-19 incidence and course in MG patients.

\section{Conclusion}

In the era of increasing availability of vaccination against COVID 19, data on unvaccinated MG patients infected with SARS-CoV-2 may contribute to a reliable assessment of the vaccination effectiveness in this group. Our study has shown that unvaccinated MG patients may experience favourable COVID-19 outcomes, even those who are immunosuppressed. Further studies are needed to evaluate international differences of COVID-19 course, taking into account the types of treatment applied and the ratio of vaccinated to unvaccinated MG patients. To the best of our knowledge, this study is the first to describe the course of COVID-19 in Polish MG patients.

Conflict of interest: None.

\section{References}

1. Gilhus NE, Tzartos S, Evoli A, et al. Myasthenia gravis. Nat Rev Dis Primers. 2019; 5(1): 30, doi: 10.1038/s41572-019-0079-y, indexed in Pubmed: 31048702.
2. Sobieszczuk E, Napiórkowski $\measuredangle$, Szczudlik P, et al. Myasthenia gravis in Poland: national healthcare database epidemiological study. Neuroepidemiology. 2021 [Epub ahead of print]: 1-8, doi: 10.1159/000512973, indexed in Pubmed: 33611318.

3. Al-Bassam W, Kubicki M, Bailey M, et al. Characteristics, incidence, and outcome of patients admitted to the intensive care unit with myasthenia gravis. J Crit Care. 2018; 45: 90-94, doi: 10.1016/j. jcrc.2018.01.003, indexed in Pubmed: 29413729.

4. Martins MM, Prata-Barbosa A, Magalhães-Barbosa MC, et al. Clinical and laboratory characteristics of SARS-COV-2 infection in children and adolescents. Rev Paul Pediatr. 2020; 39: e2020231, doi: 10.1590/19840462/2021/39/2020231, indexed in Pubmed: 33206842.

5. Wnuk M, Sawczyńska K, Kęsek T, et al. Neurological symptoms in hospitalised patients with COVID-19 and their association with inhospital mortality. Neurol Neurochir Pol. 2021; 55(3): 314-321, doi: 10.5603/PJNNS.a2021.0039, indexed in Pubmed: 34037979.

6. Finsterer J, Scorza FA, Scorza CA. Diagnosing myasthenic crisis in SARS-CoV-2 infected patients requires adherence to appropriate criteria. J Neurol Sci. 2020; 417: 117062, doi: 10.1016/j. jns.2020.117062, indexed in Pubmed: 32741592.

7. Zielińska-Turek J, Jasińska A, Kołakowska J, et al. Clinical features of neurological patients with coronavirus 2019: an observational study of one centre. Neurol Neurochir Pol. 2021; 55(2): 195-201, doi: 10.5603/PJNNS.a2021.0011, indexed in Pubmed: 33528832.

8. Gujski M, Raciborski F, Jankowski M, et al. Epidemiological analysis of the first 1389 cases of COVID-19 in Poland: A preliminary report. Med Sci Monit. 2020; 26: e924702, doi: 10.12659/MSM.924702, indexed in Pubmed: 32379726.

9. Finsterer J, Scorza FA. Perspectives of neuro-COVID: Myasthenia. Front Neurol. 2021; 12: 635747, doi: 10.3389/fneur.2021.635747, indexed in Pubmed: 33732209.

10. Singh S, Govindarajan R. COVID-19 and generalized Myasthenia Gravis exacerbation: A case report. Clin Neurol Neurosurg. 2020; 196: 106045, doi: 10.1016/j.clineuro.2020.106045, indexed in Pubmed: 32634699.

11. Ramaswamy SB, Govindarajan R. COVID-19 in refractory Myasthenia Gravis- A case report of successful outcome. J Neuromuscul Dis. 2020; 7(3): 361-364, doi: 10.3233/JND-200520, indexed in Pubmed: 32508329.

12. Sorci G, Faivre B, Morand S. Explaining among-country variation in COVID-19 case fatality rate. Sci Rep. 2020; 10(1): 18909, doi: 10.1038/s41598-020-75848-2, indexed in Pubmed: 33144595.

13. Burra P, Soto-Díaz K, Chalen I, et al. Temperature and latitude correlate with SARS-CoV-2 epidemiological variables but not with genomic change worldwide. Evol Bioinform Online. 2021; 17: 1176934321989695, doi: 10.1177/1176934321989695, indexed in Pubmed: 33551640.

14. Jaretzki A, Barohn RJ, Ernstoff RM, et al. Myasthenia gravis: recommendations for clinical research standards. Task Force of the Medical Scientific Advisory Board of the Myasthenia Gravis Foundation of America. Neurology. 2000; 55(1): 16-23, doi: 10.1212/ wnl.55.1.16, indexed in Pubmed: 10891897.

15. Wendell LC, Levine JM. Myasthenic crisis. Neurohospitalist. 2011; 1(1): 16-22, doi: $10.1177 / 1941875210382918$, indexed in Pubmed: 23983833.

16. Sanders DB, Wolfe GI, Narayanaswami P, et al. International consensus guidance for management of myasthenia gravis: Executive summary. Neurology. 2016; 87(4): 419-425, doi: 10.1212/WNL.0000000000002790, indexed in Pubmed: 27358333. 
17. Cascella M, Rajnik M, Cuomo A, et al. Features, Evaluation, and Treatment of Coronavirus (COVID-19) [Updated 2021 Mar 1]. In: StatPearls [Internet]. Treasure Island (FL): StatPearls Publishing; 2021 Jan-. https://www.ncbi.nlm.nih.gov/books/NBK554776/.

18. Słyk S, Domitrz I. Neurological manifestations of SARS-CoV-2 -a systematic review. Neurol Neurochir Pol. 2020; 54(5): 378383, doi: 10.5603/PJNNS.a2020.0050, indexed in Pubmed: 32667047.

19. Farsalinos K, Niaura R, Le Houezec J, et al. Editorial: Nicotine and SARS-CoV-2: COVID-19 may be a disease of the nicotinic cholinergic system. Toxicol Rep. 2020; 7: 658-663, doi: 10.1016/j. toxrep.2020.04.012, indexed in Pubmed: 32355638.

20. Octaviana F, Yugo HP, Safri AY, et al. Case series: COVID-19 in patients with mild to moderate myasthenia gravis in a National Referral Hospital in Indonesia. eNeurologicalSci. 2021; 23: 100332, doi: 10.1016/j.ensci.2021.100332, indexed in Pubmed: 33644426.

21. Anand P, Slama MCC, Kaku M, et al. COVID-19 in patients with myasthenia gravis. Muscle Nerve. 2020; 62(2): 254-258, doi: 10.1002/ mus.26918, indexed in Pubmed: 32392389.
22. Muppidi S, Guptill J, Jacob S, et al. COVID-19-associated risks and effects in myasthenia gravis (CARE-MG). The Lancet Neurology. 2020; 19(12): 970-971, doi: 10.1016/s1474-4422(20)30413-0.

23. Rein N, Haham N, Orenbuch-Harroch E, et al. Description of 3 patients with myasthenia gravis and COVID-19. J Neurol Sci. 2020; 417: 117053, doi: 10.1016/j.jns.2020.117053, indexed in Pubmed: 32731059.

24. Solé G, Mathis S, Friedman D, et al. Impact of coronavirus disease 2019 in a French cohort of Myasthenia Gravis. Neurology. 2021; 96(16): e2109-e2120, doi: 10.1212/WNL.0000000000011669, indexed in Pubmed: 33568541.

25. Jacob S, Muppidi S, Guidon A, et al. International MG/COVID-19 Working Group. Guidance for the management of myasthenia gravis (MG) and Lambert-Eaton myasthenic syndrome (LEMS) during the COVID-19 pandemic. J Neurol Sci. 2020; 412: 116803, doi: 10.1016/j. jns.2020.116803, indexed in Pubmed: 32247193.

26. Rzepiński Ł, Wawrzyniak S, Maciejek Z. Immunocompromised myasthenia gravis patient not infected with SARS-CoV-2 after close exposure - what is the risk of COVID-19? Neurol Neurochir Pol. 2020; 54(5): 481-482, doi: 10.5603/PJNNS.a2020.0072, indexed in Pubmed: 33006133. 